\title{
On weak regularity requirements of the relaxation modulus in viscoelasticity
}

\author{
Sandra Carillo ${ }^{1,2 *}$, Michel Chipot ${ }^{3,1}$, Vanda Valente ${ }^{4}$, Giorgio Vergara Caffarelli ${ }^{1}$ \\ ${ }^{1}$ Dipartimento di Scienze di Base e Applicate per l'Ingegneria, Università di Roma LA SAPIEnZA, \\ Via Antonio Scarpa 16, 00161 Rome, Italy \\ ${ }^{2}$ I.N.F.N. - Sezione Roma1, Gr. IV - Mathematical Methods of NonLinear Physics (M.M.N.L.P.), Rome, Italy \\ ${ }^{3}$ IMath, University of Zürich, Winterthurerstrasse 190, 8057, Zürich, Switzerland \\ ${ }^{4}$ Istituto per le Applicazioni del Calcolo M. Picone, Via dei Taurini 19, 00185 Roma, Italy \\ *Email address for correspondence: sandra.carillo@uniroma1.it \\ Communicated by Gianni Pagnini \\ Received on 11 16, 2018. Accepted on 04 24, 2019.
}

\begin{abstract}
The existence and uniqueness of solution to a one-dimensional hyperbolic integro-differential problem arising in viscoelasticity is here considered. The kernel, in the linear viscoelasticity equation, represents the relaxation function which is characteristic of the considered material. Specifically, the case of a kernel, which does not satisfy the classical regularity requirements is analysed. This choice is suggested by applications according to the literature to model a wider variety of materials. A notable example of kernel, not satisfying the classical regularity requirements, is represented by a wedge continuous function. Indeed, the linear integro-differential viscoelasticity equation, characterised by a suitable wedge continuous relaxation function, is shown to give the classical linear wave equation via a limit procedure.

Keywords: Materials with memory; Viscoelasticity; hyperbolic integro-differential problem; Regular kernel integro-differential systems; Singular kernel integro-differential systems

AMS subject classification: 74H20, 35Q74, 45K05, 74D05
\end{abstract}

\section{Introduction}

\subsection{General framework of the problem}

The mathematical model of viscoelasticity aims to take into account the behaviour of those materials whose mechanical behaviour is determined not only by the present but also on its past history. Those materials whose mechanical and/or thermodynamical response depends significantly on their past history are termed materials with memory according to the literature, see $[18,19]$ where the physical interpretation of the model as well as its thermodynamical admissibility are studied. Since then a wide variety of results and applications have been obtained in many different fields. Among the many, referring to the model of viscoelastic body, to give a feeling of the many different areas in which this model turns out to be of interest, we mention some bio-mechanical applications. In [25] synthetic tissues which mimic human bones are investigated. Indeed, the viscoelastic model is applied to a variety of biologically inspired tissues; as an example, in [25] bones, while in [24] cardiological tissues are considered. A model of apples regarded as viscoelastic bodies is studied in [23,29]. The present investigation concerns viscoelastic bodies and their mechanical behaviour aiming to widen the range of applicative cases the theory can be applied to. Accordingly, following the spirit of previous investigations, further generalisations of the classical model, suggested by applicative examples, and not previously considered, are addressed to.

The present investigation is part of a research project whose interest is focussed on materials with memory and, on one hand their mechanical or thermodynamical characterisation $[1,2]$, on the other one to study of assigned initial boundary value problems to answer to the question of existence and, possibly, uniqueness of the admitted solution. Under this viewpoint, the growing applicative interest on new 
materials, both artificial, such as polymers, and/or materials of biological origin, requires to adapt and, possibly, consider less restrictive assumptions when the mathematical model is constructed. Accordingly, when a materials characterized by mechanical response which is modeled by a Volterra type integrodifferential equation, is studied generalized kernels need to be considered. Thus, in [7] a one-dimensional viscoelasticity problem characterised by a singular kernel is studied and, later, extended in $[8,9]$.

The novelty of the present investigation consists in new relaxed requirements imposed on the kernel of the integro-differential viscoelasticity problem. Specifically, inspired by $[27,28]$, relaxations functions exhibiting a jump discontinuity in their first derivative are considered.

\subsection{Mathematical model of the problem}

The one-dimensional problem is investigated. Accordingly, the linear integro-differential equation which models the displacement response in a one dimensional viscoelastic body, whose configuration is denoted as $\Omega=(a, b) \subset \mathbb{R}$, can be written as

$$
u_{t t}=G(0) u_{x x}+\int_{0}^{t} \dot{G}(t-\tau) u_{x x}(\tau) d \tau+f
$$

where $u$ indicates the displacement while the history of the material as well as an external force, if present, are included in the term $f$. The initial boundary value problem is assigned when the following initial and boundary conditions

$$
\left.u\right|_{t=0}=u_{0}(x),\left.\quad u_{t}\right|_{t=0}=u_{1}(x),\left.\quad u\right|_{\partial \Omega \times(0, T)}=0, \quad t<T
$$

are imposed. The classical model (see for instance [14,15]), when a one-dimensional isotropic and homogeneous viscoelastic body is considered, prescribes that the kernel $G$ satisfies the conditions:

$$
\dot{G} \in L^{1}\left(\mathbb{R}^{+}\right), G(t)=G_{0}+\int_{0}^{t} \dot{G}(s) d s, G(\infty)=\lim _{t \rightarrow \infty} G(t)
$$

where $G(\infty)>0$; the fading memory property is also enjoyed, that is

$$
\forall \epsilon>0 \quad \exists \tilde{a}=a\left(\epsilon, E^{t}\right) \in \mathbb{R}^{+} \text {s.t. } \forall a>\tilde{a},\left|\int_{0}^{\infty} \dot{G}(s+a) E^{t}(s) d s\right|<\epsilon,
$$

where $E^{t}(\tau):=E(t-\tau)$ denotes the strain past history of the material. The mild formulation of the problem (1)- (2) reads

$$
u(t)=\int_{0}^{t} K(t-\tau) u_{x x}(\tau) d \tau+u_{1} t+u_{0}+\int_{0}^{t} d \tau \int_{0}^{\tau} f(\xi) d \xi,
$$

where $K$ is termed integrated relaxation function since it denotes the integral of the relaxation function defined as

$$
K(\xi):=\int_{0}^{\xi} G(\tau) d \tau
$$

Now, the relaxation function $G(t)$ is assumed to satisfy the relaxed conditions

$$
\forall t \in(0, \infty), \quad G(t)>0, \quad G \text { is non-increasing and convex. }
$$

In particular, we impose that the relaxation function $G$ is nonincreasing and convex since these conditions are induced by the adopted physical model $[19,20]$. In the present study, the choice to adopt (4) as the model equation to study the viscoelasticity problem allows to impose weaker requirements on the 
relaxation function $G$ aiming to model a wider class of materials. Thus, one of the novelties is that the function $G$ is assumed to be

$$
G \in C^{0}[0, T] \quad \forall T \in \mathbb{R},
$$

but no requirement is imposed on its time derivatives. Note that, consequently the results are referring to convex relaxation functions, which may exhibit jump discontinuities in the time derivative $\dot{G}$, according to an example, presented in [27], where the initial value $G(0)$ of the relaxation function is finite. Furthermore, the case $\dot{G}$ unbounded at $t=0$ may be considered.

The generalisation (7) is introduced to describe viscoelastic materials which are not included in previous studies in [7] wherein the relaxation function is $G \in L^{1}(0, T) \cap C^{2}(0, T), \forall T \in \mathbb{R}$, but may be unbounded at $t=0$.

\subsection{A notable example}

A special case is considered in this Subsection to stress the interest of the case under investigation. Let

$$
G(s)= \begin{cases}\frac{G_{\infty}-G_{0}}{a} s+G_{0} & 0 \leq s \leq a \\ G_{\infty} & s \geq a\end{cases}
$$

as depicted in the following Fig. 1. The evolution equation (1)

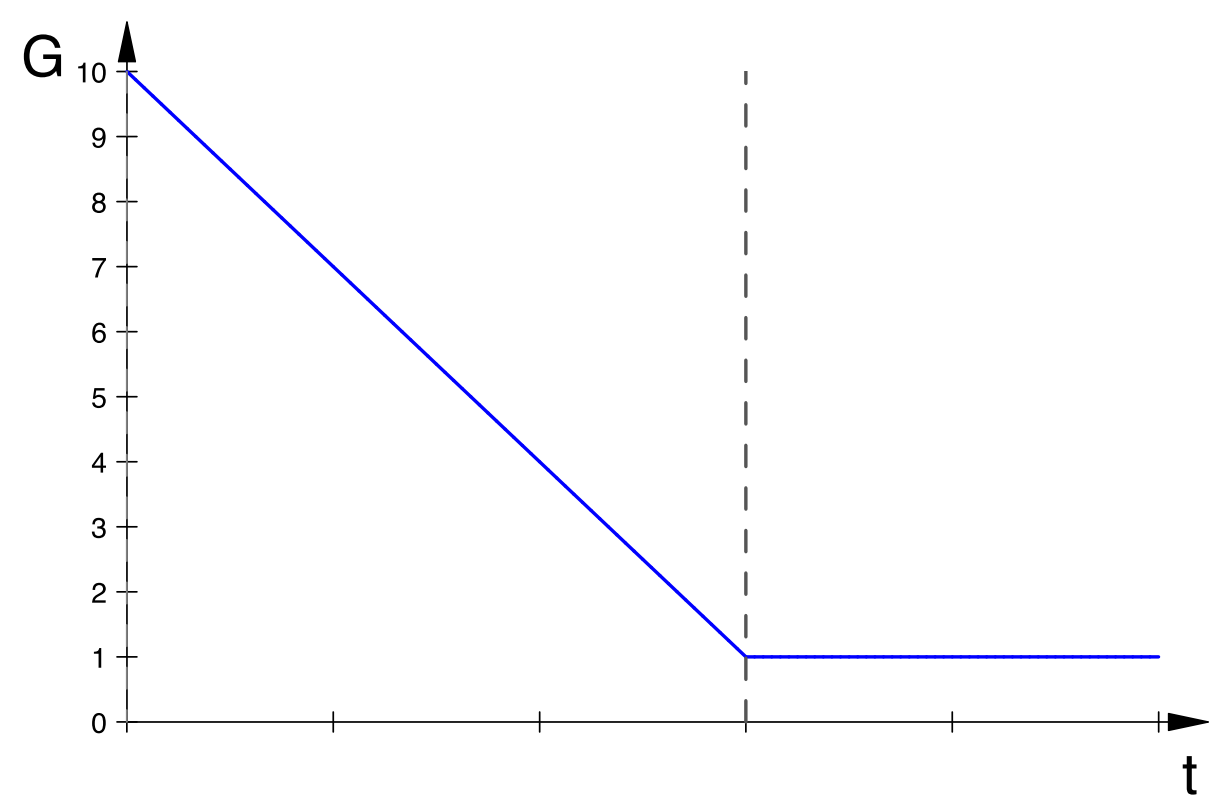

Figure 1. Relaxation function

$$
u_{t t}=G(0) u_{x x}+\int_{0}^{t} \dot{G}(t-\tau) u_{x x}(\tau) d \tau+f
$$

now, on substitution of the given relaxation function $G$, reads

$$
u_{t t}=G(0) u_{x x}+\left[G_{\infty}-G_{0}\right] \int_{\tilde{t}}^{t} \frac{1}{a} u_{x x}(s) d s+f, \quad \text { where } \quad \tilde{t}:=\max \{0, t-a\}
$$

that is, when $a \rightarrow 0, t-a>0$ definitely. Hence, on application of the mean value theorem, the limit $a \rightarrow 0$ gives

$$
u_{t t}=G(0) u_{x x}+\left[G_{\infty}-G_{0}\right] u_{x x}+f
$$


i.e.

$$
u_{t t}=G_{\infty} u_{x x}+f
$$

where $G_{0}$ does not appear since the two terms cancel out and, hence, the linear wave equation is obtained. This example shows the relation between linear wave equation and the linear viscoelasticity equation. Specifically, when we consider the wedge continuous relaxation function in the example, the linear viscoelasticity equation admits the linear wave equation as its limit wherein the speed is $c^{2}=G_{\infty}$.

\subsection{Content outline}

The outline of the article is as follows. In Section 2, a suitable mollified formulation of the relaxation function is introduced, then corresponding approximated regular problems, which depend on a small parameter $\varepsilon \in \mathbb{R}^{+}$, are considered. The two key ingredients are, on one hand, the link between the assigned initial boundary value problem (1) - (2) and its integral mild formulation (4). On the other hand, we prove that the limit, as $\varepsilon \rightarrow 0$, of the sequence of solutions admitted by the approximated regular problems coincides with the solution of the non-regular initial boundary value problem under investigation. Suitable a priori estimates are proved: the proof of existence of the weak solution admitted by the problem under investigation, and constructed via limit process, is based on them.

The subsequent Sections 3 and 4, are devoted, in turn, to show the existence, and its uniqueness, of the weak solution admitted by the problem. Sections 3 is concerned about the limit of the sequence of solutions to the approximated problems. A key role is played by the estimate in Section 2. The proof of the uniqueness of the weak solution provided in Section 3 is given in Section 4.

Perspectives and open problems are mentioned in the closing Section 5.

\section{Approximated problems}

This Section opens with the introduction of a mollified relaxation function which depends on a small parameter $\varepsilon \in \mathbb{R}^{+}$. Corresponding to each value of the parameter $\varepsilon$, a regular kernel problem is written. Thus, a sequence of approximated regular problems is constructed. On application of a Lemma, proved in [7], suitable a priori estimates are obtained. First of all, we introduce

$$
G^{\varepsilon}(t):=\int_{t-\varepsilon}^{t+\varepsilon} \rho\left(\frac{t-\tau}{\varepsilon}\right) \frac{1}{\varepsilon} G(\varepsilon+\tau) d \tau
$$

where $\rho: \mathbb{R} \rightarrow \mathbb{R}^{+}$is a $C^{\infty}$-function with compact support in the unit intervall $(-1,1)$, such that its integral is equal to one, that is

$$
\int_{\mathbb{R}} \rho\left(\frac{t-\tau}{\varepsilon}\right) \frac{1}{\varepsilon} d \tau=1
$$

so that (13) can be rewritten as

$$
G^{\varepsilon}(t):=\int_{\mathbb{R}} \rho\left(\frac{t-\tau}{\varepsilon}\right) \frac{1}{\varepsilon} G(\varepsilon+\tau) d \tau
$$

Note that by (13) one has also

$$
G^{\varepsilon}(t):=\int_{-\varepsilon}^{\varepsilon} \rho\left(\frac{\tau}{\varepsilon}\right) \frac{1}{\varepsilon} G(\varepsilon+t-\tau) d \tau
$$

so that if $G$ is nonnegative, nonincreasing and convex so is $G^{\varepsilon}$. Furthermore, formula (13) is a convolution with a mollifying kernel. Since $G$ is defined only for positive values, it is slightly extended at the left of 0 for the convolution to make sense. The chosen is the most simple natural extension. 
Now, consider the approximated problem $P^{\varepsilon}$ given by the following integro-differential equation

$$
u_{t t}^{\varepsilon}=G^{\varepsilon}(0) u_{x x}^{\varepsilon}+\int_{0}^{t} \dot{G}^{\varepsilon}(t-\tau) u_{x x}^{\varepsilon}(\tau) d \tau+f, \quad 0<\varepsilon \ll 1,
$$

where $G^{\varepsilon}$ is given by (13) and, hence, $G^{\varepsilon}(0)$ as well as $\dot{G}^{\varepsilon}$ are well defined, together with associated initial and boundary conditions

$$
\left.u^{\varepsilon}\right|_{t=0}=u_{0},\left.\quad u_{t}^{\varepsilon}\right|_{t=0}=u_{1}(x),\left.\quad u^{\varepsilon}\right|_{\partial \Omega \times(0, T)}=0 \quad, \quad t<T .
$$

Notably, the problem $P^{\varepsilon}$ is regular and, hence, admits a unique solution [14,15]. In addition, the corresponding weak formulation is:

$$
u^{\varepsilon}(t)=\int_{0}^{t} K^{\varepsilon}(t-\tau) u_{x x}^{\varepsilon}(\tau) d \tau+u_{1} t+u_{0}+\int_{0}^{t} d \tau \int_{0}^{\tau} f(\xi) d \xi
$$

where

$$
K^{\varepsilon}(\xi):=\int_{0}^{\xi} G^{\varepsilon}(\tau) d \tau
$$

The next Section is devoted to consider the limit, as $\varepsilon \rightarrow 0$ of such an approximated integral problem to the integral problem (4).

The next aim of this Section is to establish an estimate which allows to prove the needed convergence of the approximated solutions as $\varepsilon$ goes to zero.

The following Lemma 2.1 provides the needed estimates to prove the Theorem, provided in the subsequent Section, which allows us to establish the existence result we aimed to.

As proved in [14], [15], the regular linear problem (17) - (18), where $G^{\varepsilon}$ is given in (13), admits a unique solution. In particular, the following Lemma can be stated.

Lemma 2.1 Let $u^{\varepsilon}$ denote the unique solution admitted by the problem (17)-(18), then it follows

$$
\begin{gathered}
\frac{1}{2} \frac{d}{d t} \int_{\Omega} G^{\varepsilon}(t)\left|u_{x}^{\varepsilon}\right|^{2} d x-\frac{1}{2} \frac{d}{d t} \int_{0}^{t} d s \int_{\Omega} \dot{G}^{\varepsilon}(s)\left|u_{x}^{\varepsilon}(t)-u_{x}^{\varepsilon}(t-s)\right|^{2} d x+ \\
+\frac{1}{2} \frac{d}{d t} \int_{\Omega}\left|u_{t}^{\varepsilon}\right|^{2} d x=\int_{\Omega} f u_{t}^{\varepsilon} d x+\frac{1}{2} \int_{\Omega} \dot{G}^{\varepsilon}(t)\left|u_{x}^{\varepsilon}\right|^{2} d x+ \\
-\frac{1}{2} \int_{0}^{t} d s \int_{\Omega} \ddot{G}^{\varepsilon}(s)\left|u_{x}^{\varepsilon}(t)-u_{x}^{\varepsilon}(t-s)\right|^{2} d x .
\end{gathered}
$$

Proof. The proof follows the lines of the proof of Lemma 2.1 in [7].

Specifically, when we recall the conditions (6), imposed on $G$, and we consider $G^{\varepsilon}$, they imply $\dot{G}^{\varepsilon}<0$ and $\ddot{G}^{\varepsilon}>0$, in the integration time interval $(0, t)$. Hence, these conditions, combined with integration over the time interval $(0, t)$, leads to

$$
\begin{aligned}
& \frac{1}{2} \int_{\Omega} G^{\varepsilon}(t)\left|u_{x}\right|^{2} d x+\frac{1}{2} \int_{\Omega}\left|u_{t}\right|^{2} d x \\
& \leq \int_{\Omega} \int_{0}^{t} f u_{t} d x d s+\frac{1}{2} \int_{\Omega} G^{\varepsilon}(0)\left|u_{x}(0)\right|^{2} d x+\frac{1}{2} \int_{\Omega}\left|u_{1}\right|^{2} d x .
\end{aligned}
$$

The latter, when the initial data are taken into account, implies

$$
\frac{1}{2} \int_{\Omega} G^{\varepsilon}(t)\left|u_{x}\right|^{2} d x+\frac{1}{2} \int_{\Omega}\left|u_{t}\right|^{2} d x-\int_{0}^{t} \int_{\Omega}\left|u_{t}\right|^{2} d x d s \leq C\left(f, u_{1}, u_{0}, G_{0}\right) .
$$

Hence, on application of Gronwall's lemma,

$$
\frac{1}{2} \int_{\Omega} G^{\varepsilon}(t)\left|u_{x}\right|^{2} d x+\frac{1}{2} \int_{\Omega}\left|u_{t}\right|^{2} d x \leq e^{T} C\left(f, u_{1}, u_{0}, G_{0}\right) .
$$


Since $G$ is nonincreasing, choosing $2 \varepsilon \leq 1, t \leq T$ and using (14) one has

$$
G^{\varepsilon}(t) \geq \int_{t-\varepsilon}^{t+\varepsilon} \rho\left(\frac{t-\tau}{\varepsilon}\right) \frac{1}{\varepsilon} G(2 \varepsilon+t) d \tau \geq G(1+T) .
$$

Thus it holds

$$
\frac{1}{2} \int_{\Omega}\left|u_{x}\right|^{2} d x+\frac{1}{2} \int_{\Omega}\left|u_{t}\right|^{2} d x \leq \alpha e^{T} C\left(f, u_{1}, u_{0}\right),
$$

wherein $\alpha=\max \left\{(G(T+1))^{-1}, 1\right\}$. This last estimate is required later on.

\section{Integral Problem. Existence of weak solutions}

The aim of this Section is to prove that, when we let $\varepsilon \rightarrow 0$, there exists a weak limit, say $u$, of any sequence of solutions $u^{\varepsilon}$ of the approximated problem, $P^{\varepsilon}(19)$. That is, consider any sequence of functions $u^{\varepsilon}$, solutions to (19), it weakly converges to $u$, the solution to (1) - (2). The key fact is the regularity of the problem $P^{\varepsilon}$ which implies the existence and uniqueness of its solution $u^{\varepsilon}$. Then, on use of the estimate (26), it follows that there exists a subsequence $\left\{\varepsilon_{h}\right\}, h \in \mathbb{N}$ such that there exists a convergent subsequence of solutions $\left\{u^{\varepsilon_{h}}\right\}$

$$
\begin{aligned}
& u^{\varepsilon_{h}} \longrightarrow u \text { weakly in } H^{1}\left(0, T, H_{0}^{1}(\Omega)\right) \text { as } \varepsilon_{h} \rightarrow 0 ; \\
& u^{\varepsilon_{h}} \longrightarrow u \text { strongly in } L^{2}(D) \text { as } \varepsilon_{h} \rightarrow 0 ;
\end{aligned}
$$

hence,

$$
\exists u(t)=\lim _{\varepsilon_{h} \rightarrow 0} u^{\varepsilon_{h}}(t) \quad \text { in } L^{2}((a, b) \times(0, T)),
$$

where $D=(a, b) \times(0, T)$ and $u^{\varepsilon_{h}}$ is solution to the problem (17) - (18). Thus, the following result can be proved.

Theorem 3.1. The integral problem (4) admits a weak solution represented by the function $u(t)=\lim _{\varepsilon_{h} \rightarrow 0} u^{\varepsilon_{h}}(t)$.

Proof.. Consider the approximated problem (17) - (18), which admits the unique solution $u^{\varepsilon_{h}}$; the associated weak formulation reads

$$
P^{\varepsilon_{h}}: \quad u^{\varepsilon_{h}}(t)=\int_{0}^{t} K^{\varepsilon_{h}}(t-\tau) u_{x x}^{\varepsilon_{h}}(\tau) d \tau+u_{1} t+u_{0}+\int_{0}^{t} d \tau \int_{0}^{\tau} f(\xi) d \xi
$$

where

$$
K^{\varepsilon_{h}}(\xi):=\int_{0}^{\xi} G^{\varepsilon_{h}}(\tau) d \tau
$$

Then, introduce the test functions $\varphi$, which depend on both the time and space variables and satisfy homogeneous b.c.s at $a$ and $b$, i.e., at the boundaries of the $\Omega \subset \mathbb{R}$ domain

$$
\varphi \in C^{\infty}(D), \quad D=(a, b) \times(0, T), \text { s.t. } \varphi(a, t)=\varphi(b, t)=0 \quad \forall t \in(0, T) .
$$

On integration over $D$, after multiplication by $\varphi$ of (30), allows to write

$$
\begin{array}{r}
\iint_{D} u^{\varepsilon_{h}}(t) \varphi d x d t=\iint_{D} \varphi\left\{\int_{0}^{t} K^{\varepsilon_{h}}(t-\tau) u_{x x}^{\varepsilon_{h}}(\tau) d \tau+u_{1} t\right. \\
\left.+u_{0}+\int_{0}^{t} d \tau \int_{0}^{\tau} f(\xi) d \xi\right\} d x d t .
\end{array}
$$


First of all, note that all the terms

$$
\iint_{D} \varphi\left\{u_{1} t+u_{0}+\int_{0}^{t} d \tau \int_{0}^{\tau} f(\xi) d \xi\right\} d x d t
$$

depend only on the initial data and/or on the history of the material with memory, supposed known. Since all these terms are assumed regular, then the boundedness of $D$, implies the integral over $D$, in (34) is well defined and finite. Furthermore, in (34), $\varepsilon_{h}$ does not appear, hence, all the terms therein are unchanged in the limit $\varepsilon_{h} \rightarrow 0$.

Accordingly, the quantity to focuss the attention on is the following

$$
\iint_{D} \varphi d x d t \int_{0}^{t} K^{\varepsilon_{h}}(t-\tau) u_{x x}^{\varepsilon_{h}}(\tau) d \tau .
$$

On use of the homogeneous boundary conditions (32) satisfied by the test functions $\varphi$, integration by parts with respect to the space variable, two times, gives :

$$
\begin{gathered}
\iint_{D} \varphi d x d t \int_{0}^{t} K^{\varepsilon_{h}}(t-\tau) u_{x x}^{\varepsilon_{h}}(\tau) d \tau=\iint_{D} \varphi d x d t \frac{d}{d x} \int_{0}^{t} K^{\varepsilon_{h}}(t-\tau) u_{x}^{\varepsilon_{h}}(\tau) d \tau . \\
=-\iint_{D} \varphi_{x} d x d t \int_{0}^{t} K^{\varepsilon_{h}}(t-\tau) u_{x}^{\varepsilon_{h}}(\tau) d \tau
\end{gathered}
$$

The weak convergence of $u_{x}^{\varepsilon_{h}}$ to $u_{x}$ combined with the pointwise convergence of $K^{\varepsilon_{h}}$ to $K$, proved as follows, imply the existence of the weak solution. Indeed, by definition

$$
\begin{gathered}
K^{\varepsilon_{h}}(t)-K(t)=\int_{0}^{t} G^{\varepsilon_{h}}(s) d s-\int_{0}^{t} G(s) d s= \\
\int_{0}^{t} d s\left\{\int_{s-\varepsilon}^{s+\varepsilon} \rho\left(\frac{s-\tau}{\varepsilon}\right) \frac{1}{\varepsilon} G(\varepsilon+\tau) d \tau-G(s)\right\}= \\
\int_{0}^{t} d s \int_{s-\varepsilon}^{s+\varepsilon} d \tau\left\{\rho\left(\frac{s-\tau}{\varepsilon}\right) \frac{1}{\varepsilon}[G(\varepsilon+\tau)-G(s)]\right\} \rightarrow 0, \text { as } \varepsilon \rightarrow 0 .
\end{gathered}
$$

since $\lim _{\varepsilon \rightarrow 0}[G(\varepsilon+\tau)-G(s)]=0$.

\section{Integral Problem. Uniqueness of weak solutions}

In this Section the solution uniqueness is considered.

Theorem 4.1. Given the integral problem (4), i.e.,

$$
u(t)=\int_{0}^{t} K(t-\tau) u_{x x}(\tau) d \tau+u_{1} t+\int_{0}^{t} d \tau \int_{0}^{\tau} f(\xi) d \xi
$$

it admits a unique solution $u \in L^{2}\left(0, T ; H_{0}^{1}(\Omega)\right)$.

Proof.. Assume (38) admits two different solutions, say $v$ and $\tilde{v}$, then the linearity implies that also any linear combination of them is again a solution to (38). Thus, consider $w:=v-\tilde{v}$; it turns out to solve

$$
w(t)=\int_{0}^{t} K(t-\tau) w_{x x}(\tau) d \tau
$$

subject to homogeneous initial and boundary conditions, by definition. 
Let us denote by $w^{i}, \lambda_{i}$ the eigenfunctions and eigenvalues for the Dirichlet problem in $\Omega$ i.e. satisfying

$$
-w_{x x}^{i}=\lambda_{i} w^{i} \text { in } \Omega, \quad w^{i}=0 \text { on } \partial \Omega .
$$

(Without lost of generality we can assume the eigenfunctions normalised). From (39), multiplying by $w^{i}$ and integrating on $\Omega$ one deduces

$$
\int_{\Omega} w(t) w^{i} d x=-\int_{0}^{t} K(t-\tau) \int_{\Omega} w_{x}(\tau) w_{x}^{i} d x d \tau
$$

from which we derive by (40) and denoting the $L^{2}$-scalar product by $($,

$$
\left(w(t), w^{i}\right)=-\lambda_{i} \int_{0}^{t} K(t-\tau)\left(w(\tau), w^{i}\right) d \tau .
$$

Taking absolute values it follows since $K$ is nondecreasing, nonnegative

$$
\left|\left(w(t), w^{i}\right)\right| \leq K(T) \int_{0}^{t}\left|\left(w(\tau), w^{i}\right)\right| d \tau .
$$

So that by Gronwall's Lemma $\left(w(t), w^{i}\right)=0 \forall i$ and thus $w=0$.

\section{Perspectives and open problems}

The question which remains to answer to is whether it is possible to extend the obtained results to the case when a magneto-viscoelastic body is considered. Indeed, the case of magneto-viscoelastic materials is very interesting both under the applicative viewpoint as well as under the mathematical one. Specifically, the parabolic equation that describes the evolution of the magnetisation spin is modified by the introduction of a further term when the deformation effect is taken into account. There are many publications on the subject, among them we quote the modelling of magneto-elastic interactions described in [4], and the variational analysis and some aspects on the modelling and numerics considered in [17,22]. When the mechanical response also accounts of the past deformation, the mathematical problem becomes an evolution problem with memory described by nonlinear integro-differential equations. In the framework of the model proposed in [3], the problem, when the two effects elastic and magnetic effects are coupled is studied in $[12,13,26]$. One of the aspects is the change from parabolic to hyperbolic of the system, studied also in [16]. The case of a magneto-viscoelastic material where equations which couple the two effects of viscoelasticity and magnetisation are given, in [5,6]. In [5] we prove the existence and uniqueness of the solution admitted by a 1-dimensional magneto-viscoelastic problem. The existence result is generalised to the 3-dimensional case in [6] and to the case of a singular kernel 1-dimensional magneto-viscoelastic problem in [10]. Singular kernel problems are studied also in $[8,11]$. Currently under investigation is the coupling of the magnetic effects with the viscoelasticity model considered in the present study.

\section{Acknowledgments.}

The authors gratefully acknowledge the partial financial support of the Italian G.N.F.M.-I.N.d.A.M. and of Università di Roma LA SAPIEnZA. S. Carillo thanks also I.N.F.N.-Sez. Roma1, Gr. M.M.N.L.P.; M. Chipot thanks the Italian G.N.F.M.-I.N.d.A.M. and Dipartimento di Scienze di Base e Applicate per l'Ingegneria, Università di Roma LA SAPIENZA, for the kind hospitality.

\section{References}

1. G. Amendola, S. Carillo and A. Manes, Classical free energies of a heat conductor with memory and the minimum free energy for its discrete spectrum model, Bollettino U. M.l., sect. B, vol.3, pp. 421-446, 2010. 
2. G. Amendola, S. Carillo, J.M. Golden and A. Manes, Viscoelastic fluids: free energies, differential problems and asymptotic behaviour, Discrete and Continuous Dynamical Systems - Series B, vol. 19, pp.1815-1835, 2014.

3. M. Bertsch, P. Podio-Guidugli and V. Valente, On the dynamics of deformable ferromagnets, I. Global weak solutions for soft ferromagnets at rest, Ann. Mat. Pura Appl. (IV), vol. CLXXIX, pp. 331-360, 2001.

4. W.F. Brown, Magnetoelastic Interactions, Springer Tracts in Natural Philosophy, vol.9, Springer Verlag, 1966.

5. S. Carillo, V. Valente, and G. Vergara Caffarelli, A result of existence and uniqueness for an integrodifferential system in magneto-viscoelasticity, Applicable Analisys, vol. 90, pp. 1791-1802, 2010.

6. S. Carillo, V. Valente, and G. Vergara Caffarelli, An existence theorem for the magnetic-viscoelastic problem, Discrete and Continuous Dynamical Systems Series S., vol. 5, pp. 435-447, 2012.

7. S. Carillo, V. Valente and G. Vergara Caffarelli, A linear viscoelasticity problem with a singular memory kernel: an existence and uniqueness result, Differential and Integral Equations, vol. 26, pp. 1115-1125, 2013.

8. S. Carillo, Singular kernel problems in materials with memory, Meccanica, vol. 50, pp. 603-615, 2015.

9. S. Carillo, A 3-dimensional singular kernel problem in viscoelasticity: an existence result, Atti della Accademia Peloritana dei Pericolanti, Classe di Scienze Fisiche, Matematiche e Naturali, vol. 97 (S1), 13 pp., 2019. DOI: 10.1478/AAPP.97S1A3.

10. S. Carillo, M. Chipot, V. Valente and G. Vergara Caffarelli, A magneto-viscoelasticity problem with a singular memory kernel, Nonlinear Analysis Series B: Real World Applications, vol. 35C, pp. 200-210, 2017.

11. S. Carillo, V. Valente and G. Vergara Caffarelli, Heat conduction with memory: a singular kernel problem, Evolution Equations and Control Theory, vol. 3, pp. 399-410, 2014.

12. M. Chipot, I. Shafrir, V. Valente, and G. Vergara Caffarelli, A nonlocal problem arising in the study of magneto-elastic interactions, Boll. UMI Serie IX, I, pp. 197-222, 2008.

13. M. Chipot, I. Shafrir, V. Valente, and G. Vergara Caffarelli, On a hyperbolic-parabolic system arising in magnetoelasticity, J. Math. Anal. Appl., vol. 352, pp. 120-131, 2009.

14. C.M. Dafermos, An abstract Volterra equation with applications to linear viscoelasticity, J. Diff. Equations, vol. 7, pp. 554-569, 1970.

15. C.M. Dafermos, Asymptotic stability in viscoelasticity, Arch. Rat. Mech. Anal.,vol. 37, pp. 297-308, 1970.

16. M. De Angelis, On the transition from parabolicity to hyperbolicity for a nonlinear equation under Neumann boundary conditions, Meccanica, Article in Press, 2018.

17. A. DeSimone and G. Dolzmann, Existence of minimizers for a variational problem in two-dimensional nonlinear magneto-elasticity, Arch. Rational Mech. Anal., vol. 144, pp. 107-120, 1998.

18. M. Fabrizio, G. Gentili and J.M. Golden, Non-isothermal free energies for linear theories with memory, Mathematical and Computer Modelling, vol. 39, pp. 219-253, 2004.

19. M. Fabrizio and A. Morro, Mathematical problems in linear viscoelasticity, SIAM Studies in Applied Mathematics, 12, Philadelphia, PA, 1992.

20. G. Gentili, Maximum recoverable work, minimum free energy and state space in linear viscoelasticity, Quart. Appl. Math., vol. 60, pp. 153-182, 2002.

21. C. Giorgi and A. Morro, Viscoelastic solids with unbounded relaxation function, Continuum Mechanics And Thermodynamics, vol. 4, pp. 151-165, 1992.

22. D. Kinderlehrer, Magnetoelastic interactions, in variational methods for discontinuous structures, 
Prog. Nonlinear Differential Equations Appl., vol. 25, , Birkhauser Basel, pp. 177-189, 1996.

23. Md. Mahiuddin, Md. Imran H. Khan, Nghia Duc Pham and M.A. Karim, Development of fractional viscoelastic model for characterising viscoelastic properties of food material during drying, Food Bioscience, vol.23, pp. 45-53, 2018.

24. A. Rassoli, N. Fatouraee and R. Guidoin, Structural model for viscoelastic properties of pericardial bioprosthetic valves, Artificial Organs, vol. 42, pp. 630-639, 2018.

25. A. Shahin-Shamsabadi, et al., Mechanical, material, and biological study of a PCL/bioactive glass bone scaffold: Importance of viscoelasticity, Materials Science and Engineering: C, vol. 90, pp. 280$288,2018$.

26. V. Valente and G. Vergara Caffarelli, On the dynamics of magneto-elastic interactions: existence of solutions and limit behavior, Asymptotic Analysis, vol. 51, pp. 319-333, 2007.

27. G. Vergara Caffarelli, Dissipativity and uniqueness for the one-dimensional dynamical problem of linear viscoelasticity (Italian), Atti Accad. Naz. Lincei Rend. Cl. Sci. Fis. Mat. Natur., vol. 83, pp.483488, 1988.

28. G. Vergara Caffarelli, Dissipativity and existence for the one-dimensional dynamical problem of linear viscoelasticity (Italian), Atti Accad. Naz. Lincei Rend. Cl. Sci. Fis. Mat. Natur., vol. 82, pp. 489-496, 1988.

29. Z. Stropek and K. Golacki, Viscoelastic response of apple flesh in a wide range of mechanical loading rates, International Agrophysics, vol. 32, pp. 335-340, 2018. 